\title{
CURVILINEAR RECONSTRUCTIONS FOR EBR SCHEMES ON SEMI-STRUCTURED MESHES
}

\author{
PAVEL V. RODIONOV ${ }^{1}$ \\ ${ }^{1}$ Keldysh Institute of Applied Mathematics \\ Miusskaya Sq. 4, Moscow, 125047, Russia \\ pavel.rodionov.cs@gmail.com
}

Key words: Semi-structured Mesh, EBR Scheme, Curvilinear Reconstruction, Boundary Layer.

\begin{abstract}
The vertex-centered edge-based reconstruction (EBR) schemes for solving Eulertype equations are originally developed for tetrahedral unstructured meshes and imply the usage of quasi-one-dimensional straight-line reconstructions to calculate the flux. However, on the hybrid unstructured meshes with layers of highly anisotropic prismatic cells that are commonly used in simulations of turbulent flows with boundary layers, the straight-line reconstructions may lead to strongly irregular stencils, which cause both larger approximation errors and computational instabilities. To improve the accuracy and stability characteristics of EBR schemes on such meshes, we propose to use the curvilinear reconstructions for the flux computation. In this paper, we describe the principles and algorithms of constructing curvilinear reconstructions on the structured and semi-structured prismatic meshes. The modified EBR schemes are tested on the well-known NACA 0012 airfoil validation case for both two-dimensional and three-dimensional formulations, and the obtained numerical results are discussed in detail.
\end{abstract}

\section{INTRODUCTION}

The correctness of turbulent flow simulations essentially relies on accuracy of the boundary layers resolving. In practice, an acceptable accuracy cannot be achieved without constructing meshes with very fine resolution in the wall-normal direction, which usually leads to the appearance of highly anisotropic cells near the streamlined body. Such cells form structured or semi-structured prismatic layers in the boundary layer region and their geometry could be utilized to improve accuracy of the methods based on wide stencils.

In this study, we consider the vertex-centered edge-based reconstruction (EBR) schemes that were initially formulated for unstructured tetrahedral meshes [1]. These schemes are based on quasi-one-dimensional reconstructions and employ wide stencils to provide the higher accuracy. The original formulation of EBR schemes implies the use of straight lines as the location domain of reconstruction points. However, on the curvilinear meshes with highly anisotropic cells the application of straight-line reconstructions may lead to higher solution error and cause stability issues. To eliminate such effects within the prismatic layers, we propose to use curvilinear reconstructions that better correspond to the boundary layer 
stratification of the physical variables. Earlier, the similar approach was implemented for the flux correction method [2,3].

The paper is organized as follows. We formulate mathematical model and the original EBR schemes in Sections 2 and 3, respectively. Then we describe the curvilinear modifications of EBR schemes for structured and semi-structured prismatic meshes in Section 4. Section 5 represents the results of validation and testing of the modified EBR schemes on NACA 0012 airfoil validation case [4] for both two and three dimensions.

\section{MATHEMATICAL MODEL}

We consider compressible Navier-Stokes equations

$$
\frac{\partial \boldsymbol{Q}}{\partial t}+\nabla \cdot F(\boldsymbol{Q})=\nabla \cdot F_{v}(\boldsymbol{Q}, \nabla \boldsymbol{Q})
$$

where

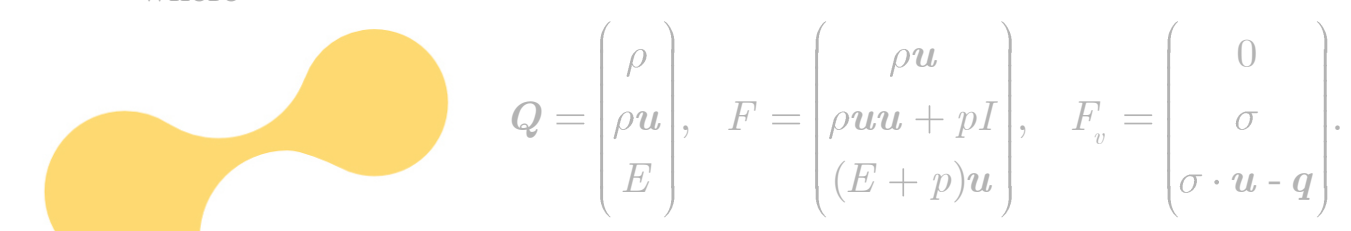

Here, $\rho$ is the density, $u$ is the velocity vector, $p$ is the pressure and $E$ is the total energy.

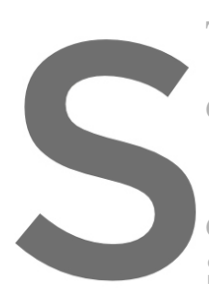
The stress tensor is defined by $\sigma=\mu\left(\nabla u+(\nabla u)^{\mathrm{T}}-(2 / 3)(\nabla \cdot u) I\right)$, where
dynamic viscosity coefficient, I denotes the identity matrix. Heat flux is int
$q=-k \nabla T$, where $k$ is the coefficient of thermal conductivity and $T$ is the temp
describe dependency of the dynamic viscosity coefficient from the temperature,
Sutherland's law.

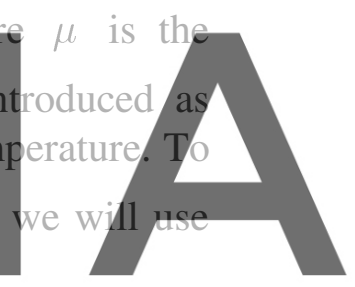

In the section devoted to validation of the new methods, the Reynolds-averaged Navier-

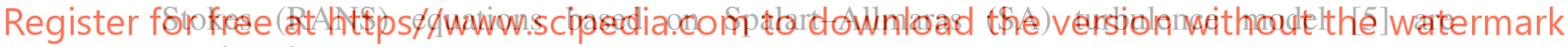
employed.

\section{EBR SCHEMES}

To solve the equations (1) numerally on a mixed-element mesh, we construct vertexcentered barycentric cells (called ordinary in [6]) around each node and define discrete nodal function $\boldsymbol{Q}_{i}$, where $i$ denotes the node index. The examples of two-dimensional vertexcentered barycentric cells are given in Figure 1. The general form of the edge-based schemes can be written as

$$
\frac{d \boldsymbol{Q}_{i}}{d t}+\frac{1}{V_{i}} \sum_{j \in N_{1}(i)} \boldsymbol{F}_{i j} s_{i j}=\boldsymbol{F}_{v, i},
$$

where $V_{i}$ is the volume of the cell corresponding to node $i, \boldsymbol{F}_{i j}$ is the approximation of the convective flux $F \cdot \boldsymbol{n}$ at the midpoint of the edge $i j, \boldsymbol{n}$ is the outer-pointing unit normal, $s_{i j}$ is the area of the common face between cells that correspond to nodes $i$ and $j, N_{1}(i)$ is the 
set of first-order neighbors of node $i$ and $\boldsymbol{F}_{v, i}$ is the approximation of the diffusive terms in node $i$. To approximate the convective flux, we use the Roe method

$$
\boldsymbol{F}_{i j}=\frac{1}{2}\left(F\left(\boldsymbol{Q}_{i j}^{R}\right)+F\left(\boldsymbol{Q}_{i j}^{L}\right)\right) \cdot \boldsymbol{n}_{i j}-\frac{1}{2}\left|A_{i j}\right|\left(\boldsymbol{Q}_{i j}^{R}-\boldsymbol{Q}_{i j}^{L}\right),
$$

where $\boldsymbol{Q}_{i j}^{L / R}=R_{i j}^{L / R}\{\boldsymbol{Q}\}, \quad R_{i j}^{L / R}\{\boldsymbol{Q}\} \quad$ are the operators of straight-line edge-based reconstruction and $\boldsymbol{n}_{i j}$ is the integral mean of normal $\boldsymbol{n}$ calculated over the common face between cells that correspond to nodes $i$ and $j$.

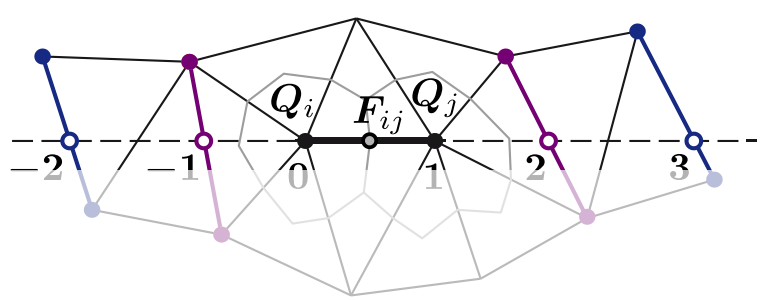

Figure 1: Stencil of the EBR5 scheme for the edge $i j$ on a triangular unstructured mesh

The algorithm of quasi-one-dimensional reconstruction used in the original EBR5 scheme is as follows [1]. For each node of the edge $i j$ we build the sets of topological neighbors of the first and second order. which nodes are the seco $j i$ with the faces of the carried out for node. representation of these
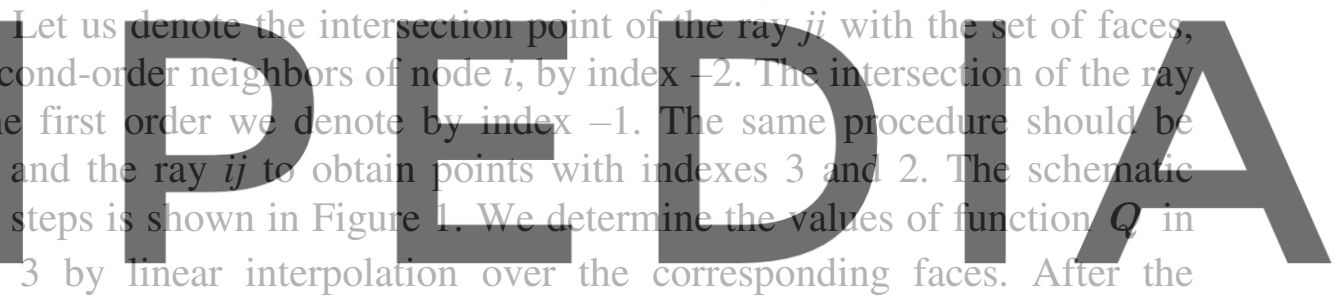

points $-2,-1,2$ and 3 by linear interpolation over the corresponding faces. After the denotation of nodes $i$ and $j$ with the indexes 0 and 1 , respectively, it becomes possible to

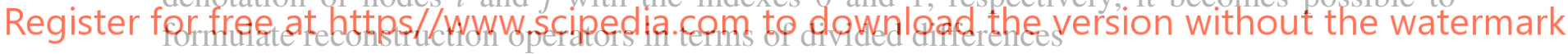

$$
\Delta_{m}^{L}\{\boldsymbol{Q}\}=\frac{Q_{m+1}-Q_{m}}{\left|\mathbf{r}_{m+1}-\mathbf{r}_{m}\right|}, \quad \Delta_{m}^{R}\{\boldsymbol{Q}\}=\Delta_{-m}^{L}\{\boldsymbol{Q}\}
$$

as

$$
\begin{aligned}
& R_{i j}^{L}\{\boldsymbol{Q}\}=\boldsymbol{Q}_{i}+\frac{\left|\mathbf{r}_{i}-\mathbf{r}_{j}\right|}{2} \sum_{m} \beta_{m} \Delta_{m}^{L}\{\boldsymbol{Q}\}, \\
& R_{i j}^{R}\{\boldsymbol{Q}\}=\boldsymbol{Q}_{j}-\frac{\left|\mathbf{r}_{i}-\mathbf{r}_{j}\right|}{2} \sum_{m} \beta_{m} \Delta_{m}^{R}\{\boldsymbol{Q}\},
\end{aligned}
$$

where $\beta_{-2}=-1 / 15, \beta_{-1}=11 / 30, \beta_{0}=4 / 5, \beta_{1}=-1 / 10$ [1]. For the EBR3, which requires shorter stencil, the corresponding coefficients are $\beta_{-2}=0, \beta_{-1}=1 / 3, \beta_{0}=2 / 3, \beta_{1}=0$. On uniform meshes in the case of linear equations with constant coefficients, the EBR5 provides the fifth order accuracy, the EBR3 - the third order. 


\section{EBR SCHEMES WITH CURVILINEAR RECONSTRUCTIONS}

Straight-line reconstructions may be applied on arbitrary unstructured mesh by the definition. However, the accuracy of such reconstructions is directly related to the specific mesh structure. Especially it concerns to highly anisotropic meshes in boundary layer regions, where straight-line reconstructions can significantly increase numerical error and cause computation instabilities. The two main reasons of these effects are illustrated in Figure 2. The first is the imbalance of the distances within reconstruction stencil, which potentially leads to the instability of the numerical solution because even slight curvature of regular anisotropic mesh is interpreted as a tremendous mesh irregularity by the method. The second is the location of reconstruction points that is not consentient with high-gradient flow inside the boundary layer and therefore causes increased variation of the physical variables within the stencil resulting in higher numerical error.

To eliminate these disadvantages, we propose to replace straight-line reconstructions in the EBR schemes by the reconstructions based on curvilinear stencils.

\subsection{Structured meshes}

The formulation of curvilinear reconstructions is especially simple and straightforward in the case of structured meshes. For these meshes, as shown in Figure 2, it is enough to consider topologically neighboring nodes $-2 \mathrm{~S},-1 \mathrm{~S}, 2 \mathrm{~S}$ and $3 \mathrm{~S}$ as the reconstruction points and build the corresponding reconstructions without any modification of formulas (2). Resulting stencil provides the better distance balance and reduces the variation of physical variables. Hereinafter the EBR schemes were already

Here, we also note that reconstructions may lead to even greater error, e. corners. To avoid this behavior, it is enough to limit the deviation of angle within

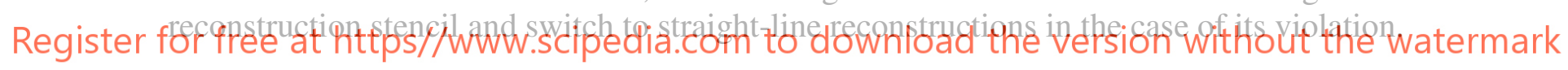

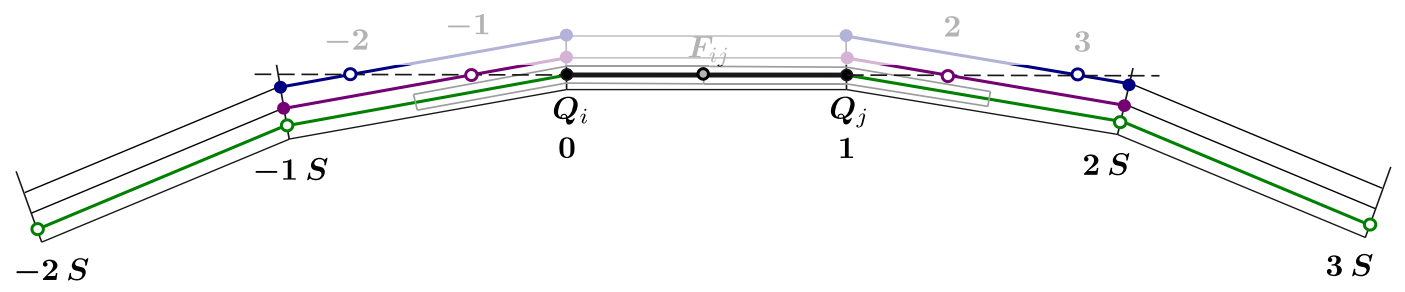

Figure 2: Stencils of the EBR5 and EBR5 IJK schemes for the edge $i j$ on an anisotropic structured mesh in the near-wall region

\subsection{Semi-structured prismatic meshes}

Layers of prisms, as may be seen in Figure 3, generally do not have the structure in tangential directions and therefore do not allow constructing curvilinear reconstructions by the completely structural approach.

In this paper, we propose the algorithm of creating curvilinear reconstructions that utilizes only mesh structure in the normal direction. The preliminary step required by this algorithm is the markup of all considering prismatic layers. In vertex-centered case, it can be done by 
defining for each node the minimal topological distance from the boundary. Accordingly, the nodes that lie on the streamlined surface will form the zero layer, their prismatic neighbors will form the first layer and so forth.

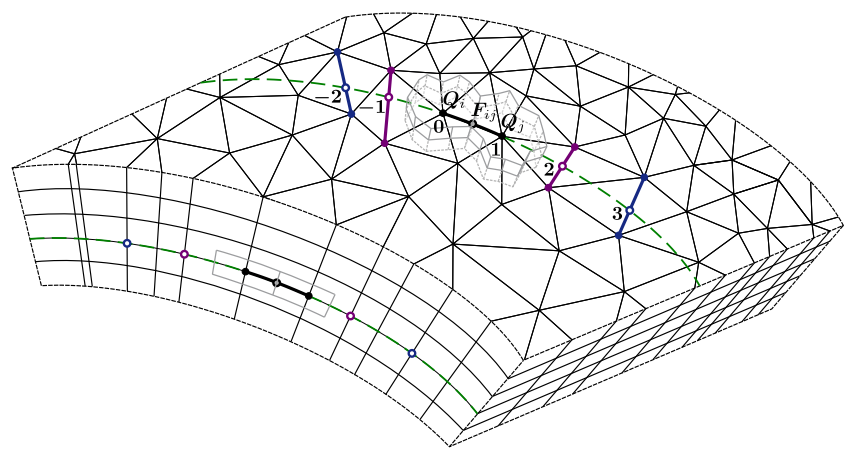

Figure 3: Stencil of the EBR5 SS scheme for the edge ij on a prismatic mesh in the near-wall region

To reconstruct conservative variables on the edge $i j$ connecting nodes $i$ and $j$ that belong to the same prismatic layer, we use the following algorithm that is illustrated in Figure 4:

1. Build the sets of first- and second-order neighbors for nodes $i$ and $j$.

2. Exclude from these sets the nodes that do not belong to the considering prismatic layer.

3. For each set of nodes, assign the set of edges, which are defined only by nodes from the

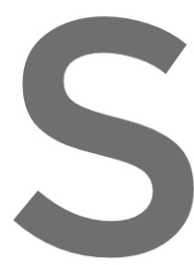
corresponding set.

4. Define the projection plane by the edge $i$

normals built to the faces incident to the edge

only one incident face, let the vector $\vec{P}$ equal
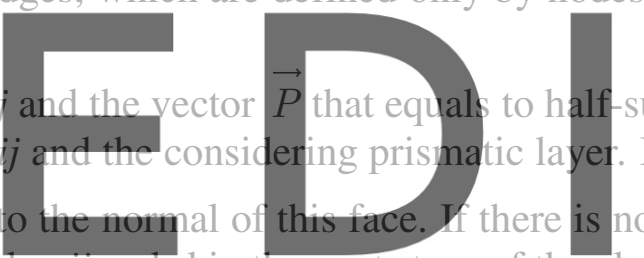

face, use straight-line reconstructions for the edge ij and skip the next steps of the algorithm.

5. Project the sets of edges obtained at Step 3 onto the plane defined by the edge ij and the

Register for free at https//Www.scipedia.com to download the version without the watermark vector $P$.

6. Determine straight-line reconstruction stencil inside the projection plane for the edge $i j$.

7. Define curvilinear reconstructions by formulas (2) using the values of metric coefficients that correspond to the preimages of obtained reconstruction points from the prismatic layer.

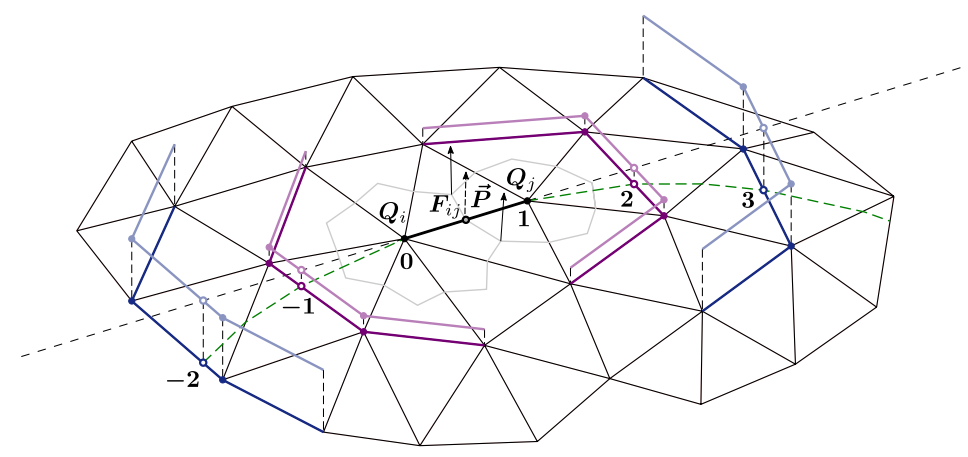

Figure 4: Algorithm of the curvilinear stencil construction for the EBR5 SS scheme 
For the edges that do not belong to any prismatic layer, we apply the straight-line reconstructions in complete accordance with formulas (2). Hereinafter we will denote the resulting scheme as EBR SS (Semi-Structured). This scheme does not have an additional computational costs in comparison with the straight-line EBR because all the described steps are supposed to be made only once before the main computation process.

\section{NUMERICAL RESULTS}

To test the curvilinear modifications of EBR schemes, we use NACA 0012 airfoil validation case [4]. The two-dimensional and three-dimensional computations of the problem are carried out by the in-house code NOISEtte [8].

\subsection{Formulation of the test case}

The infinite wing based on NACA 0012 airfoil of a unit chord is placed inside the uniform airflow with the Mach number 0.15 and the temperature $300 \mathrm{~K}$. The Reynolds number based on chord length equals to $6 \times 10^{6}$. The considered angles of attack (AOA) are $0^{\circ}$ and $10^{\circ}$.

\subsection{Computation parameters and boundary conditions}

In two-dimensional formulation, the computational domain is defined by the square $-500 \leq x / c, y / c \leq 500$ with the leading edge of airfoil placed at its center. The extrusion
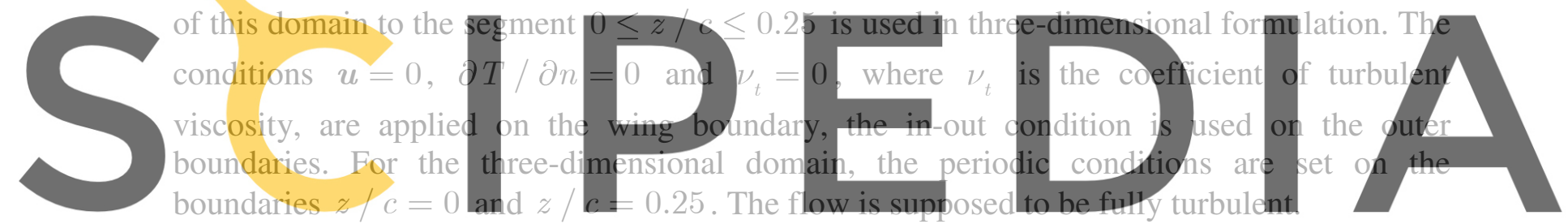

The two-dimensional computations are carried out on the sequence of hybrid meshes that

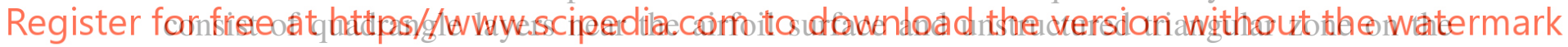
rest of the domain. Schematic representation of such meshes is given in Figure 5. The sizes of these meshes are given in Table 1, where $N$ is the total number of mesh nodes and $N_{\text {surf }}$ is the number of nodes on the airfoil surface. To validate the two-dimensional schemes, we also apply the structured mesh $897 \times 257$ that is used in [4] to obtain the reference numerical results.

The three-dimensional computations are carried out on the analogous sequence of meshes that are triangular prismatic near the wing surface and unstructured tetrahedral on the rest of the domain. These meshes coincide with the corresponding two-dimensional meshes at the cross-sections $z / c=0$ and $z / c=0.25$. The main parameters of three-dimensional meshes are given in Table 2, where $N_{\text {surf, } z=0}$ is the number of nodes on the airfoil surface at the cross-section $z / c=0$. All meshes in the present study satisfy the $y^{+}<1$ condition at the considered angles of attack.

In all computations, we use the EBR schemes to approximate the convective fluxes. The diffusive fluxes are approximated by the method of local element splittings, which is described in [9] . Pseudo-time integration is done by the implicit first-order scheme that is resolved by one Newton iteration based on BiCGSTAB method with ILU0 preconditioner. 
Computations are carried out until the convergence of total energy, turbulent viscosity, drag and lift coefficients.

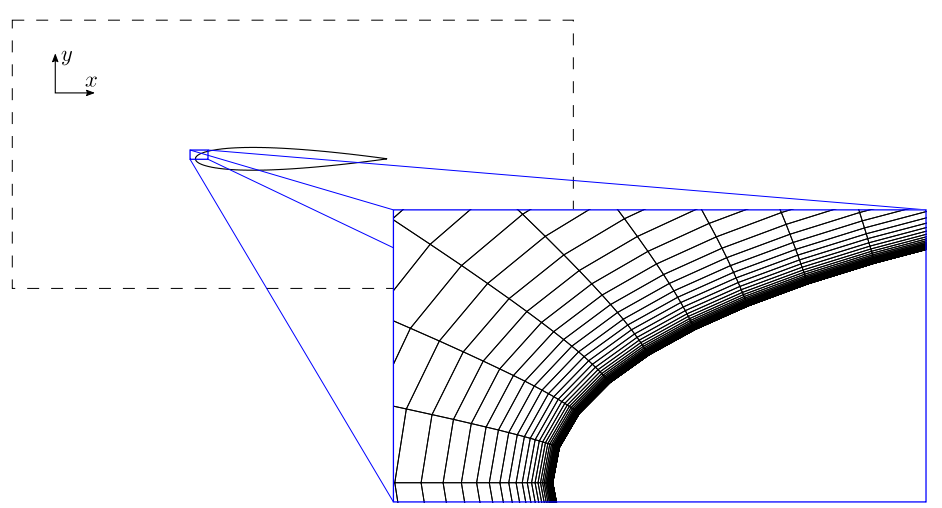

Figure 5: Configuration of the NACA 0012 meshes

Table 1: Parameters of the two-dimensional meshes

\begin{tabular}{ccccccc}
\hline $2 \mathrm{D}$ mesh & $\mathrm{x} 1$ & $\mathrm{x} 2$ & $\mathrm{x} 3$ & $\mathrm{x} 4$ & $\mathrm{x} 8$ & $897 \times 257$ \\
\hline$N$ & $55 \mathrm{~K}$ & $51 \mathrm{~K}$ & $69 \mathrm{~K}$ & $83 \mathrm{~K}$ & $84 \mathrm{~K}$ & $231 \mathrm{~K}$ \\
$N_{\text {surf }}$ & 102 & 162 & 246 & 442 & 930 & 513
\end{tabular}
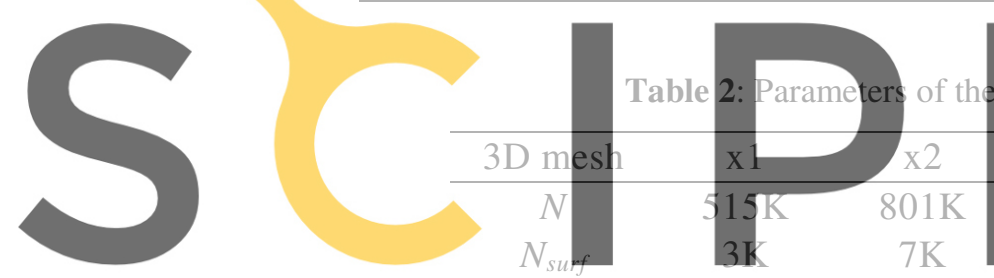

$N_{\text {surf,z=0 }} \quad 102$

162
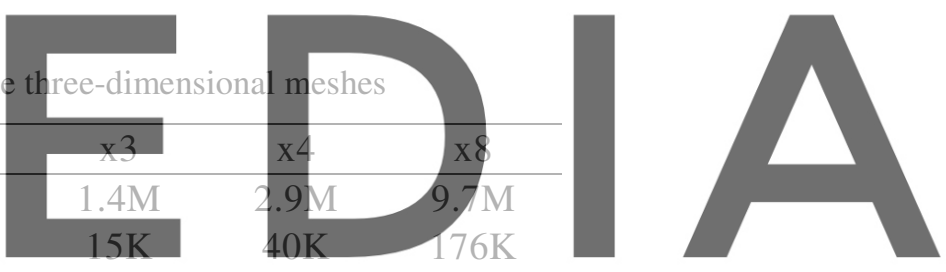

Register for free at https//www.scipedia.com to download the version without the watermark

We should note that the EBR schemes with straight-line reconstructions appear to be unstable on very coarse meshes because of the significant stencil imbalance. To increase the stability, we introduce the parameter $C_{\lim }$ that corresponds to the maximum distance ratio within the reconstruction stencil. If distances within the reconstruction stencil violate this limitation, the scheme switches to the EBR3. If violation is still present, we multiply the coefficient $\beta_{-1}$ in formulas (2) by $C_{\lim } \times\left|\mathbf{r}_{0}-\mathbf{r}_{-1}\right| /\left|\mathbf{r}_{i}-\mathbf{r}_{j}\right|$. In the current study, it is enough to use $C_{\lim }=20$ to stabilize the computation process on the very coarse meshes.

\subsection{Validation of the numerical methods}

The results obtained by the straight-line EBR5 scheme and its curvilinear modifications on the finest two-dimensional and three-dimensional hybrid meshes $x 8$ and the structured mesh $897 \times 257$ are compared with the reference experimental and numerical data from [4].

Comparison of the lift and drag coefficients is given in Table 3. We see that the deviation of obtained results from the mean reference values does not exceed $1 \%$ for the lift coefficient at $\mathrm{AOA} 10^{\circ}$ and the drag coefficient at $\mathrm{AOA} 0^{\circ}$, and does not exceed $2 \%$ for the drag 
coefficient at AOA $10^{\circ}$. We also note that on the same mesh the difference between EBR5 schemes with straight-line and curvilinear reconstructions does not exceed $0.1 \%$ for the lift coefficient and $0.4 \%$ for the drag coefficient. Distributions of the pressure and friction coefficients are shown in Figure 6. As we see, the results obtained by the EBR5 IJK and EBR5 SS agree well with the experimental data and almost coincide with the reference numerical results.

Table 3: Validation of the EBR5, EBR5 IJK and EBR5 SS schemes by the lift coefficient $\left(\mathrm{C}_{l}\right)$ and the drag coefficient $\left(\mathrm{C}_{d}\right)$

\begin{tabular}{lllcccc}
\hline Scheme & $(\mathrm{mesh})$ & $0^{\circ}: C_{l}$ & $10^{\circ}: C_{l}$ & $0^{\circ}: C_{d}$ & $10^{\circ}: C_{d}$ \\
\hline EBR5 & $(3 \mathrm{D}, \mathrm{x} 8)$ & $\sim 0$ & 1.0862 & 0.00810 & 0.01234 \\
EBR5 SS & $(3 \mathrm{D}, \mathrm{x} 8)$ & $\sim 0$ & 1.0865 & 0.00810 & 0.01233 \\
EBR5 & $(2 \mathrm{D}, \mathrm{x} 8)$ & $\sim 0$ & 1.0875 & 0.00811 & 0.01239 \\
EBR5 IJK & $(2 \mathrm{D}, \mathrm{x} 8)$ & $\sim 0$ & 1.0871 & 0.00812 & 0.01237 \\
\hline EBR5 & $(897 \times 257)$ & $\sim 0$ & 1.0946 & 0.00810 & 0.01264 \\
EBR5 IJK & $(897 \times 257)$ & $\sim 0$ & 1.0940 & 0.00810 & 0.01259 \\
\hline CFL3D & $(897 \times 257)$ & $\sim 0$ & 1.0909 & 0.00819 & 0.01231 \\
FUN3D & $(897 \times 257)$ & $\sim 0$ & 1.0983 & 0.00812 & 0.01242 \\
NTS & $(897 \times 257)$ & $\sim 0$ & 1.0891 & 0.00813 & 0.01243 \\
\hline
\end{tabular}
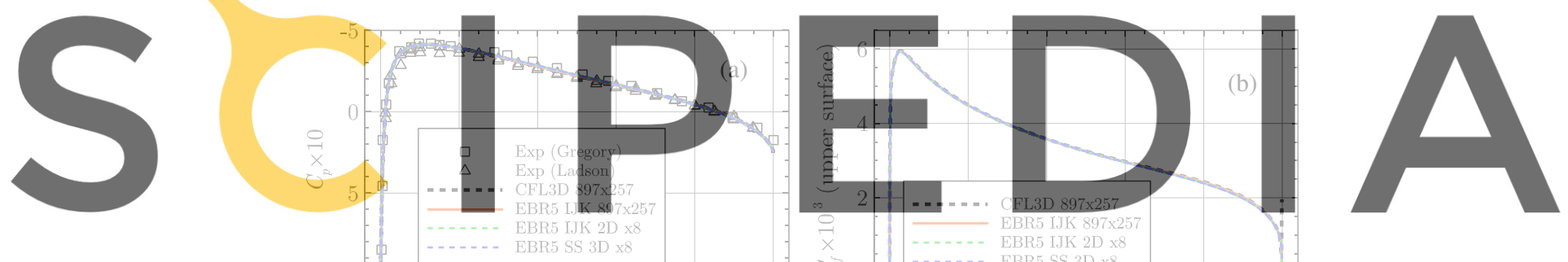

Register for free at https//wWw.scipedia.com to download the version without the watermark
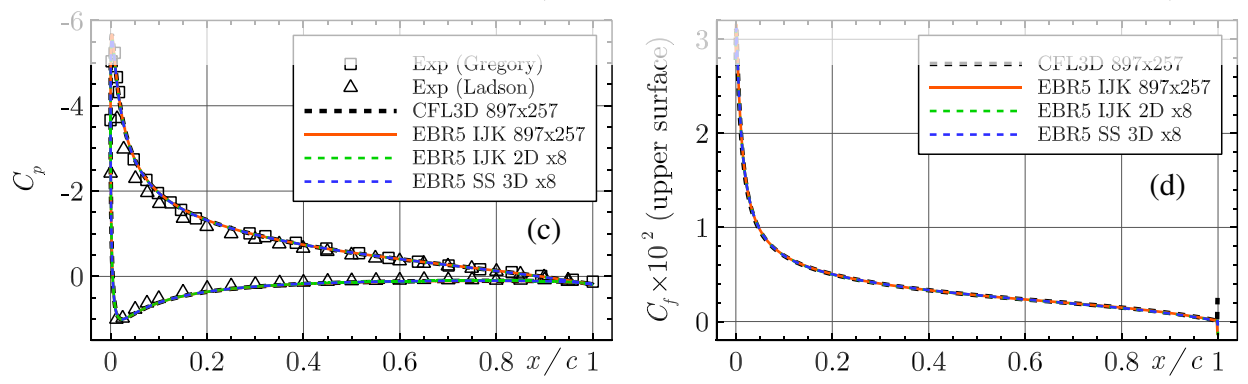

Figure 6: Validation of the EBR5 IJK and EBR5 SS schemes by the pressure coefficient $\left(C_{p}\right)$ and the friction coefficient $\left(C_{f}\right)$ at $\mathrm{AOA} 0^{\circ}(\mathrm{a}, \mathrm{b})$ and $10^{\circ}(\mathrm{c}, \mathrm{d})$

The conducted validation confirms the ability of the modified EBR schemes to simulate steady-state aerodynamic flow, as well as the correctness of their current implementation. 


\subsection{Comparison of the EBR schemes based on straight-line and curvilinear reconstructions}

To study the effect of using curvilinear reconstructions in the EBR schemes in more detail, we consider the numerical results obtained on the sequences of two-dimensional and threedimensional hybrid meshes.

The two components of the drag coefficient at AOA $0^{\circ}$ depending on the number of nodes on the airfoil surface are represented in Figure 7. The finer meshes predictably provide more accurate results, and the EBR5 shows better accuracy than the EBR3 as expected. The threedimensional results appear to be closer to the reference values than the corresponding results of two-dimensional computations, which may be explained by the differences in node density between the regular quadrangle and unstructured triangle meshes. The curvilinear reconstructions demonstrate the significant improvement of accuracy on the coarse meshes; on the fine meshes, the difference between straight-line and curvilinear reconstructions almost disappears due to the gradual straightening of the curvilinear stencils in the boundary layer. Note that on the coarsest meshes x1 the curvilinear EBR5 schemes already provide the results that are very close to the reference values from the finest meshes.
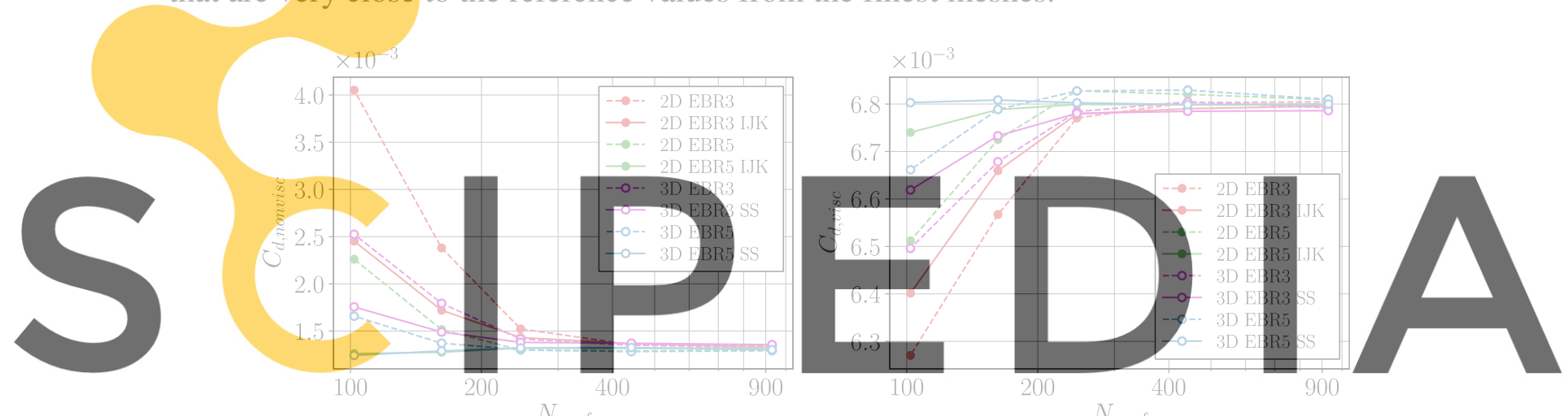

Register for free at https//www.scipedia.com to download the versjon without the watermark Figure 7: Non-viscous and viscous components of the drag coeft

The described observations remain valid at AOA $10^{\circ}$, which is confirmed by the results depicted in Figure 8. Moreover, the presented results show the excellence of the EBR3 SS not only over the straight-line EBR3 but also over the straight-line EBR5 that has wider reconstruction stencil. This once again emphasizes the benefits from using curvilinear reconstructions in the EBR schemes.

The most noticeable distributions of the pressure and friction coefficients obtained on the coarse two-dimensional meshes are presented in Figure 9. These results demonstrate the necessity of using curvilinear reconstructions on the coarse meshes, at least to get the reasonable distribution of the friction coefficient.

The spatial distributions of the friction coefficient obtained by the EBR5 schemes on the fine three-dimensional meshes are given in Figure 10. We see that the application of straightline reconstructions on such meshes causes non-physical fluctuations of the friction coefficient, which take place even on the finest mesh. It is possible to avoid these fluctuations by using the curvilinear modification of the EBR5 scheme. The same effect can be achieved 
for the EBR3 scheme and for AOA $10^{\circ}$.
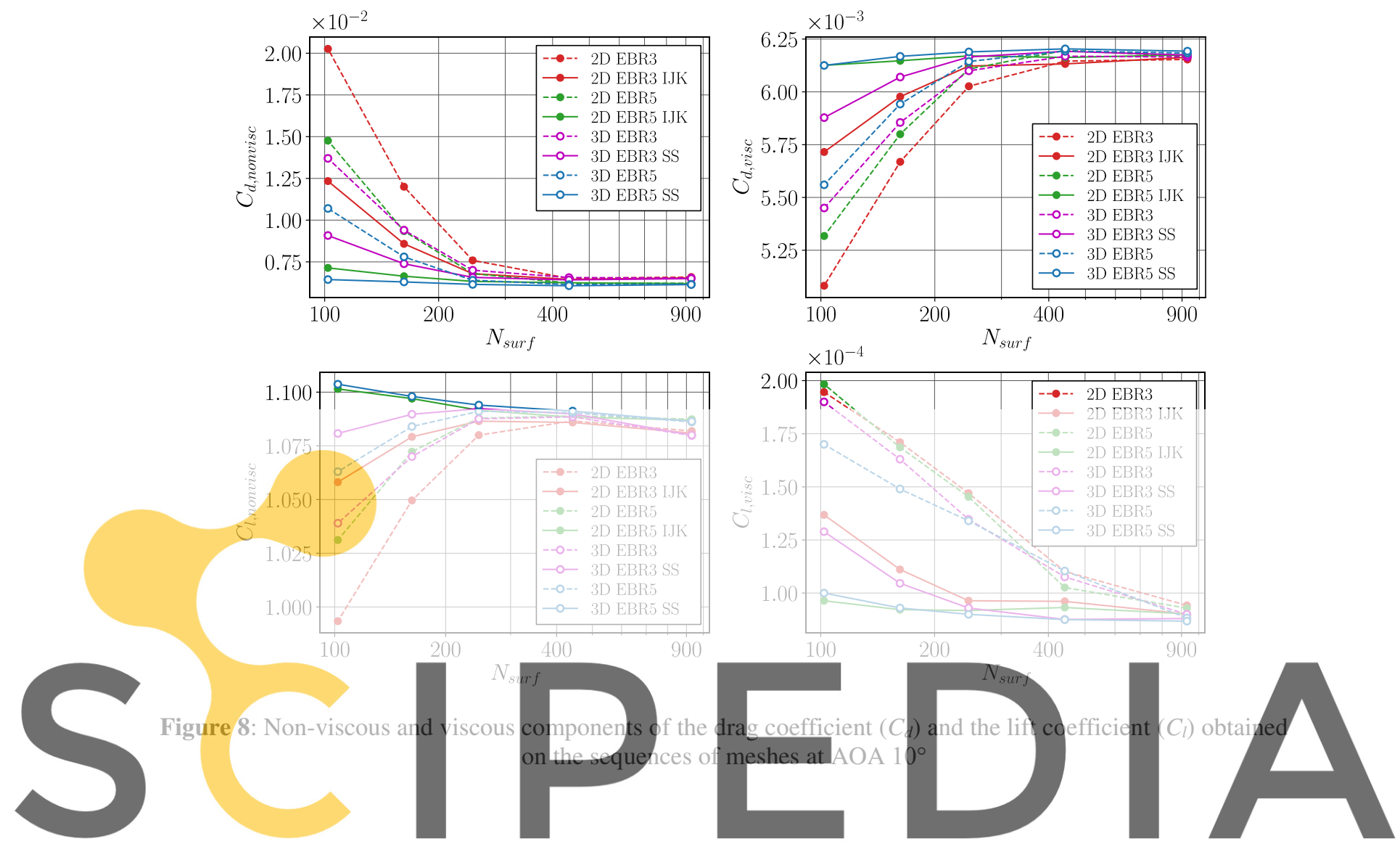

Register for free at https//WWW.scipedia.com to download the version without the watermark
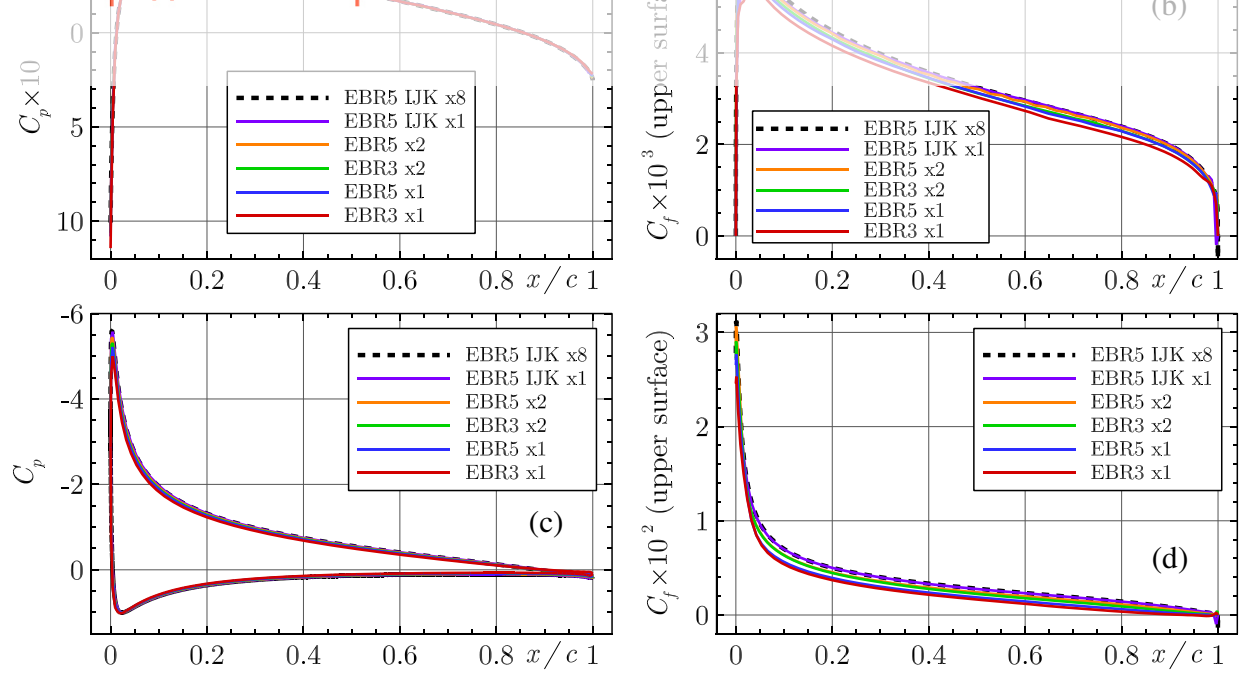

Figure 9: Most noticeable distributions of the pressure coefficient $\left(C_{p}\right)$ and the friction coefficient $\left(C_{f}\right)$ obtained on the coarse two-dimensional meshes at $\operatorname{AOA~} 0^{\circ}(\mathrm{a}, \mathrm{b})$ and $10^{\circ}(\mathrm{c}, \mathrm{d})$ 


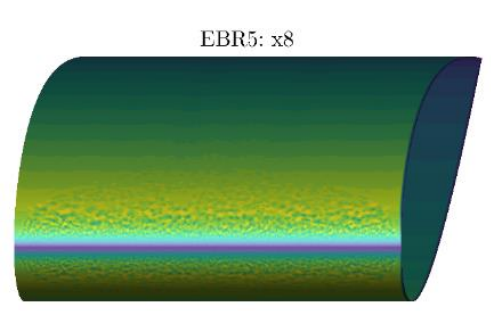

EBR5 SS: $\mathrm{x} 8$

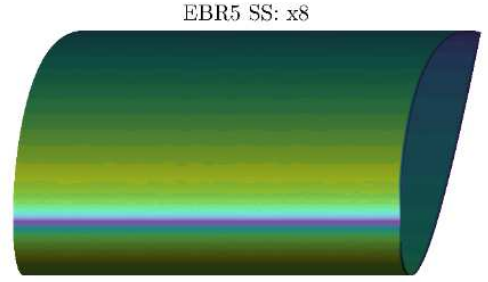

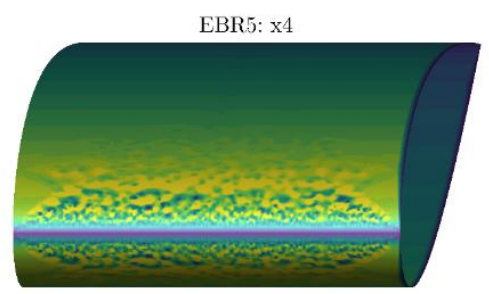

EBR5 SS: $\mathrm{x} 4$

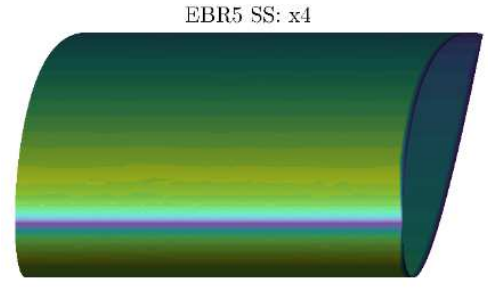

\begin{tabular}{lllllllll|l} 
& & & & & & & & & \\
$C_{f}:$ & 0 & 0.0013 & 0.0026 & 0.0039 & 0.0052 & 0.0065
\end{tabular}

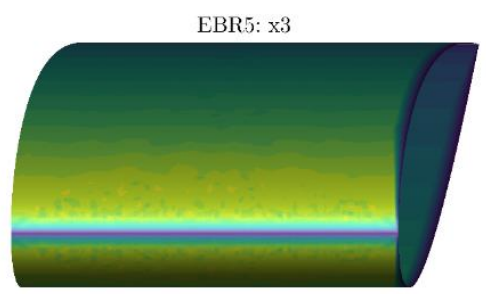

EBR5 SS: $\mathrm{x} 3$

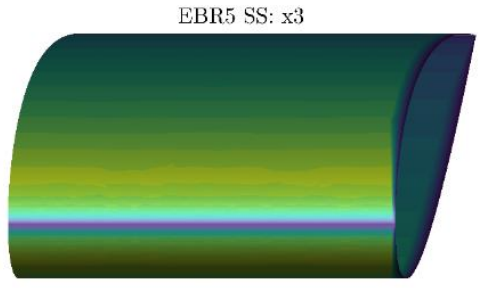

$\uparrow^{y}$

Figure 10: Friction coefficient distributions on the wing surface obtained by the EBR5 and EBR5 SS schemes on the three-dimensional meshes $\mathrm{x} 8, \mathrm{x} 4$ and $\mathrm{x} 3$ at $\mathrm{AOA} 0^{\circ}$

\section{CONCLUSIONS}

We formulated the modifications of the EBR schemes that use curvilinear reconstructions on the structured and semi-structured prismatic meshes. On the series of tests based on NACA 0012 airfoil validation case, we demonstrated the two main advantages of using these modifications in boundary layer zones. The first is the increased stability of the method provided by the better balance of distances between the reconstruction points. The second is the higher accuracy of the method achieved by decreased variation of physical variables within the reconstruction stencil due to the employment of nodes from the same stratification levels of the boundary layer. The EBR schemes with curvilinear reconstructions are proved to have the same computational performance as the original EBR schemes.

\section{ACKNOWLEDGMENTS}

The computations were performed on the hybrid supercomputer K60 installed in the Supercomputer Centre of Collective Usage of KIAM RAS with the additional use of the Shared research facilities of HPC computing resources at Lomonosov Moscow State University.

\section{REFERENCES}

[1] Abalakin I.V., Bakhvalov P.A., Kozubskaya T.K. Edge-based reconstruction schemes for unstructured tetrahedral meshes. Int. J. Numer. Methods Fluids (2016) 81(6):331-356.

[2] Katz A.J., Work D. High-order flux correction/finite difference schemes for strand grids. J. Comput. Phys. (2015) 282:360-380.

[3] Tong O., Katz A.J., Wissink A.M., Sitaraman J. High-order methods for threedimensional strand-cartesian grids. AIAA Paper 2015-0835. 53rd AIAA Aerospace 
Sciences Meeting (2015).

[4] NASA Langley Research Center. Turbulence modeling resource. 2DN00: 2D NACA 0012 airfoil validation case. URL: https://turbmodels.larc.nasa.gov/naca0012_val.html. SA model results. URL: https://turbmodels.larc.nasa.gov/naca0012_val_sa.html.

[5] Spalart P.R., Allmaras S.R. A one-equation turbulence model for aerodynamic flows. AIAA Paper 92-0439. 30th Aerospace Science Meeting (1992).

[6] Bakhvalov P.A., Kozubskaya T.K. On efficient vertex-centered schemes on hybrid unstructured meshes. AIAA Paper 2016-2966. 22nd AIAA/CEAS Aeroacoustics Conference (2016).

[7] Duben A.P., Kozubskaya T.K. On scale-resolving simulation of turbulent flows using higher-accuracy quasi-1D schemes on unstructured meshes. Progress in Hybrid RANSLES Modelling. HRLM. Notes on Numerical Fluid Mechanics and Multidisciplinary Design (2016) 137:169-178.

[8] Gorobets A. Parallel algorithm of the NOISEtte code for CFD and CAA simulations. Lobachevskii J. Math. (2018) 39(4):524-532.

[9] Bakhvalov P.A. Method of local element splittings for diffusion terms discretization in edge-bases schemes. Keldysh Institute Preprints (2020) 79:1-43 